\title{
Computer Development and Application in Network Environment
}

\author{
Yang Li \\ Yunnan College of Traditional Chinese Medicine 650200
}

Keywords: network environment, computer, C language, development application

\begin{abstract}
The development of computer technology has now been integrated into our daily lives and played a good role in the integration of various production activities and other technologies. This article will briefly discuss computer-related applications and developments, and combine information security with mobile network environments.
\end{abstract}

The development of computer technology has greatly enriched people's acceptance of daily information, but at the same time it has caused some problems in information security, such as various types of social problems caused by information security are still emerging in an endless stream. Including the future development direction of computers under the network environment, this article will normatively explain the programming aspects of the C language Knowledge, combined with past and present development trends.

\section{The key to computer development: $\mathrm{C}$ language}

\subsection{Application advantages of $C$ language}

First of all, in the use of computer programming languages, $C$ language can accurately express the data and words it contains, and its types are also varied. On the basis of the binary language, it can solve the over-abstract problem in the original programming language. Structural processing and improvement are more efficient. The large-capacity internal memory needed in the use of the $\mathrm{C}$ language and the basic requirements that can perfectly support each byte have a unique number can be basically satisfied under the current conditions, so the corresponding

The programmer can also set the variable initial value to control the overall variable effect from the memory characteristics and language data types.

And to achieve effective use of $C$ language to deal with the key links. From this we can see that the $\mathrm{C}$ language, which is the basis of programming, has obvious advantages in the improvement of the overall computer.

\subsection{C language use points}

The first part is about the basic advantages of the $\mathrm{C}$ language in terms of application. Next, it will focus on the actual application process.

A few points in the basic explanation:

The first thing to say is pointers in the $C$ language. The pointer variable will appear as a "*" before the variable declaration. The development of computer technology in the network environment is now integrated into the daily life around us and it is also plays a good role in the integration between various production activities and other technologies. This article will briefly discuss computer-related applications and developments, and combine information security with mobile network environments. Moreover, in the complete $\mathrm{C}$ language programming, generally there will not only one pointers, such as the pointer variable actually refers to the storage address of the variable, and "*" represents the storage content it contains, but also can be another A variable, array, and function address. The $\mathrm{C}$ language has a characteristic difference from it other programming languages. If the pointer is a formal parameter of the program, you can use the function and get the return value different from return(z) at the same time. It is a function unmatched by the use of pointers in other high-level programming languages. 
Second, we have to mention the string in the $C$ language, the $C$ language string is an array of characters based on the most basic text as the writing, which has the characteristics of, because the string itself is a chair Array, so its array exit form does not need to reference the library and is based on ASCII UNL.

Once again, we will talk about the structural problems of the $C$ language. We know that the most commonly used structures in the $\mathrm{C}$ language are the selection structure, the sequence structure, and the loop structure.

(1) The selection structure is a C language structure that selectively performs the basic judgments based on the relevant conditions. The various branch conditions and the analysis program flow are intertwined and combined to form the core part of the selection structure. Let's take a look at the common ones. Several choice structure statements, such as "if" and "if-else", are the most common choices for constructing statements; and statements like "switch case" begin with a multi-branch selection structure.

(2) The sequential ordering of certain rules forms the sequence structure. For example, the letters $\mathrm{X}, \mathrm{Y}, \mathrm{Z}$ are set to three values. At this time we know that $\mathrm{X}=4, \mathrm{Y}=6$, if people want to make $\mathrm{X}$ values of $\mathrm{Y}$ and $\mathrm{Y}$ are exchanged with each other, and their specific editingW

The process can be expressed as follows:

$$
\mathrm{Z}=\mathrm{X}, \quad \mathrm{X}=\mathrm{Y}, \quad \mathrm{Y}=\mathrm{Z}
$$

Then the corresponding operation result is $\mathrm{X}=6, \mathrm{Y}=\mathrm{Z}=4$ but if the order is changed, the result will be changed:

$$
\mathrm{X}=\mathrm{Y}, \quad \mathrm{Z}=\mathrm{X}, \quad \mathrm{Y}=\mathrm{Z}
$$

Then the result of the operation will change to $\mathrm{X}=\mathrm{Y}=\mathrm{Z}=6$. This is a concrete embodiment of the sequence structure. In practice, the sequential structure can help the computer to achieve a sort of combing and perfect fluency in the process of input, operation and output effect.

(3) The C language can also achieve the effect of continuously executing the same command repeatedly. This uses the loop structure, which is also called the loop body. In this way, if the programmer used to write some basic programming language repeatedly, he can use the loop. The repeated execution of the body will greatly enhance its workload and work efficiency, and will also greatly reduce the programming time of the entire program language.

\section{Computer Information Security}

Modern people's concerns about information security in the use of personal computers will, so it needs people to take corresponding measures for information security, then in the future development and application of computers, improve management awareness in all aspects.

(1) The software and hardware aspects of the computer network can be solved in most cases in response to existing system vulnerabilities and virus attacks. Therefore, the main focus now is on prevention and detection. Start with, and conduct careful research on the corresponding viruses, and be prepared to respond to the emergence of other new types of viruses. The encryption process of the program must be updated from time to time and check for vulnerabilities. At the same time, we must also do a good job in the hardware maintenance of the device. In addition to regular maintenance and repairs, we must also step up updates and introduce new types of hardware to optimize the overall technology upgrade.

(2) It is also necessary to emphasize that at least the following points should be made with regard to the safe operating environment of the network. If you are concerned about the loss of information due to power failure, you should pay attention to the maintenance work in peacetime. Whether the battery is in a normal working state and must be charged and discharged in a timely manner. The operation of the computer is to ensure that the temperature cannot be too high or too low, so the ventilation work is very necessary. If the operation is overheated, it is very easy to breakdown. In the daily use of the safety aspects of its use, we must do a good job in three areas, the first is 
whether thunderstorms do a good job of preparation measures for lightning protection, the second is the usual maintenance can guarantee that the surrounding is dry State, the third is absolutely do not expose computers to water in large areas, in order to ensure that the computer in the processing operation of a good working condition.

(3) The relevant personnel performing the operation must avoid subjective reasons of their own causing computer system problems. Therefore, the relevant management departments need to take measures for the professionalism of the operators and related computer security management personnel. In addition to intensifying organizational training and implementing corresponding rewards and penalties according to the conditions of the staff, they should be able to acquire professional knowledge for all the people can lay the foundation for the conscious cultivation of the system's safe operation and the provision of good services.

(4) The information security problem in the mobile network environment is also not to be ignored. The popularity of smart phones has made people have higher requirements on system security, but the existing environment for Android-based mobile phones uses the overall experience of the Android system. With respect to security, it is still not possible for all users to feel at ease. Because of the open nature of the Android system, it also gives the virus an opportunity. Therefore, it is still necessary to focus on strengthening this aspect.

\section{Computer Technology in Mobile Network Environment}

The first thing to say is the mobile network. The mobile network brings us convenience in life. It is self-evident.

Now it is easy for us to achieve the goal of accessing the Internet in anywhere,anytime, and obtaining information in an explosive manner.

The growth has benefited from the use of mobile networks. Basically, mobile phones can now replace the Internet search capabilities of most computers.

Energy, as well as access to information, has become the main use of Internet devices. The combination of $4 \mathrm{G}$ and Wi-Fi has also greatly facilitated communication and transaction among people, and has gradually become a major part of people's communication. Use basics, such as using WeChat and qq software, to achieve short communication, rather than talking entirely through the original communication technology.

Then, based on the popularization of mobile networks, the development of application software has also been developed, which is the result of the use of computer technology in the mobile network environment. The advantages of the application are complete and convenient and fast. The favor has even changed the lifestyle of most users. In the future there will be a lot of room for develop application softwar. The number of users will gradually increase further. Therefore, the development of information technology under the mobile network environment is still very broad and worth to waiting.

\section{The development direction of computer technology}

The R\&D and breakthroughs of various technologies are constantly refreshing our imagination. Computer technology in the mobile network environment is advancing at an especially alarming rate. At the same time, 5G mobile networks have been developed on the basis of 4G.

The network type of such high-speed transmission, if you want to achieve complete implementation and popularity, you can imagine that it can trigger new round of economic reshuffling of the IT industry is undoubtedly the inevitable development direction of information technology in the mobile network environment.

From Figure 1 we can see a gradual development curve of the computer from the beginning to the present. We can see that one of the situations that emerged was that a trend from rapid increase to gradual decrease, especially in the current position,It is said that the development of computers is now more focused on the concept of no longer an early breakthrough in the overall concept, but by its branches. 
After development, we need to break through, we need to start to improve the effectiveness of each branch in order to achieve the overall improvement.

At the surgical level, today's computer technology has developed very diversely and has produced many directions for reference.

We know that the combination of computer technology and the Internet gives us great convenience in life. In the future, we will continue to improve the ease of use of computer technology so that it can be tested and combined with a number of different technologies. We believe that Produce many unexpected effects, for example, can be mutually combined with 3D printing technology, and sales on the Internet, people around the world can even print some of their own desired through the corresponding data model stored in the computer Instruments that can be molded once, even through the corresponding design software, can be designed and adjusted according to their own preferences. This is an imaginative and practical idea for people. Well, this is just a combination of computer and a technology. If it is a combination of other technologies, it can create room for development in different industries. Even the fusion of these technologies can even produce epoch-making effects. Obviously, the development space of computer technology in various industries is still unimaginable. This also requires us to start from ourselves and constantly carry out exploration. Figure 1: The S-curve of technological development and the integration of technological aspects into the study of imagination To combine development.

\section{Concluding remarks}

In today's technological research environment, people are committed to continuously breaking through the technological breakthroughs of various types of digital devices.

The integration of computer technology, such as VR glasses, Internet smart TV, XBOX game center, etc., these new digital devices that people can directly contact with in daily life are constantly absorbing the integration of computer technology to achieve better experience. Then the relevant discussion on the basis of the past research in this paper also hopes to make a certain contribution to the development of computer technology in the future.

\section{References}

[1] Shi Jifeng. The development and application of computer technology in mobile network environment [J]. Computer fan, 2016(09):122-123.

[2] Jiang Yuhao. Analysis of Computer Development and Application under Network Environment [J]. China Newcom, 2017, 19(01):77-78.

[3] Wu Ensheng. Research on Computer Information Processing Technology Security under Network Environment [J]. Enterprise Science and Technology, 2015(08):10-11.

[4] Zhang Tao. The development and application of computer communication technology in the new era background [J]. Information Systems Engineering, 2016(11):28.

[5] Nie Jiang. The Development and Application of Computer Communication Technology in the New Times [J]. Digital Communication World, 2016(03):38-39.

[6] Wei Jingtao. Discussion on computer hardware security protection strategy under network environment [J]. Computer Knowledge and Technology, 2015(09):37-38. 